\title{
NECESSARY AND SUFFICIENT CONDITIONS FOR THE VALIDITY OF HILBERT TYPE INTEGRAL INEQUALITIES WITH A CLASS OF QUASI-HOMOGENEOUS KERNELS AND ITS APPLICATION IN OPERATOR THEORY
}

\author{
Yong Hong, Bing He AND Bicheng YANG
}

\begin{abstract}
By using real analysis technique and the method of weight functions, the necessary and sufficient conditions for the validity of Hilbert type integral inequalities with a class of quasi-homogeneous kernels and the best constant factors are obtained, and its applications in operator theory are discussed.
\end{abstract}

Mathematics subject classification (2010): 26D15, 47A07.

Keywords and phrases: Quasi-homogeneous kernel, Hilbert's integral inequality, necessary and sufficient conditions, best constant factor, bounded operator, operator norm.

\section{REFERENCES}

[1] B. He, J. F. CAO, B. C. YANG, A brand new multiple Hilbert-type integral inequality, Acta Mathematics Sinica, Chinese Series 58, 4 (2015), 661-672.

[2] Y. Hong, A Hilbert-type integral inequality with quasi-homoeneous kernel and several functions, Acta Mathematics Sinica, Chinese Series 57, 5 (2014), 833-840.

[3] I. Perić, P. Vuković, Hardy-Hilbert's inequalities with a general homogeneous kernel, Math. Inequal. Appl. 12 (2009), 525-536.

[4] M. TH. RASSIAS, B. C. YANG, On a Hardy-Hilbert-type inequality with a general homogeneous kernel, Int. J. Nonlinear Anal. Appl. 7, 1 (2016), 249-269.

[5] Q. Chen, Y. P. SHI, B. C. YANG, A relation between two simple Hardy-Mulholland-type inequalities with parameters, J. Inequal. Appl. 2016 (2016), 1-14.

[6] Y. Hong, Y. M. WEN, A necessary and sufficient condition of that Hilbert type series inequality with homogeneous kernel and the best constant factor, Chin. Ann. Math. 37A, 3 (2016), 329-336.

[7] B. C. YANG, Q. Chen, On a more accurate Hardy-Mulholland-type inequality, J. Inequal. Appl. 2016 (2016), 1-16.

[8] M. Z. GAO, B. C. YANG, On the extended Hilbert's inequality, Proc. Amer. Math. Soc. 126, 3 (1998), $751-759$.

[9] B. C. YANG, On a more accurate multidimensional Hilbert-type inequality with parameters, Math. Ineq. Appl. 18 (2015), 429-441.

[10] D. M. XIn, B. C. YANG, Q. Chen, A discrete Hilbert-type inequality in the whole plane, J. Inequal. Appl. 2016 (2016), 1-12.

[11] J. C. KUANG, Applied Inequalities, Shangdong Science and Technology Press, Jinan, 2004.

[12] B. C. YANG, Q. CHEN, On a Hardy-Hilbert-type inequality with parameters, J. Inequal. Appl. 2015 (2015), 1-18.

[13] B. C. YAnG, Q. Chen, A new extension of Hardy-Hilbert's inequality in the whole plane, J. Funct. Space 2016 (2016), Art. ID 9197476, 1-8.

[14] Y. Hong, On multiple Hardy-Hilbert integral inequalities with some parameters, J. Inequal. Appl. 2006 (2006), Art. ID 94960, 1-11.

[15] Q. L. HUANG, B. C. YANG. On a multiple Hilbert-type integral operator and applications, J. Inequal. Appl. 2009 (2009), Art. ID 192197, 1-13. 
[16] B. C. YANG, Q. CHEN, A more accurate multidimensional Hardy-Hilbert-type inequality with a general homogeneous kernel, J. Math. Inequal. 12, 1 (2018), 113-128.

[17] G. H. Hardy, J. E. Littlewood, G. Pólya, Inequalities, Gambridge University Press, London, 1952. 